\title{
Contact Area and Temperature of Same-Parameter Fractal Surfaces in Sliding Process
}

\author{
Liu Bingbing ${ }^{1}$, Zhou Chao ${ }^{1, a}$ \\ ${ }^{1}$ Fuzhou University in Fuzhou, CHINA \\ ${ }^{2}$ Fuzhou University, CHINA
}

\begin{abstract}
To evaluate the ability of fractal parameters for characterizing tribological properties of surfaces, some stable fractal surfaces, which possess the same fractal dimension, scale factor and root-mean-square deviation of surface topography, were synthesized, and then simulated using finite element analysis to calculate the maximum temperature and contact area during sliding process. It was found that for same-parameter fractal surfaces the maximum temperature and contact area fluctuated dramatically. Thus, the tribological properties of surfaces can not be characterized by some simple parameters.
\end{abstract}

\section{Introduction}

Tribology, which plays a more and more important role in mechanic engineering under the rapid development of science and technology in the 21st century, focuses on the phenomena occurred on the surface of components such as friction, wear, lubrication, etc. Since real surfaces are rough, so many studies were dedicated to the surface modeling or the prediction of surface roughness $[1,2]$. With the insight of previous studies [3], the surfaces of mechanical elements possess the geometric properties of self-similarity and selfaffinity, which makes fractal turn into one of the most popular modeling methods for rough surfaces since it is able to characterize those properties in all scales with two essential parameters, fractal dimension (D) and scale factor (C). For example, OZER, et al [4] performed a thermo-mechanical contact analysis of the magnetic head-disk interface using fractal surfaces, and VALLET, et al [5] validated the use of synthesized fractal surfaces in their work. Traditional surface characteristic parameters such as root-mean-square (RMS) deviation of surface topography (Sq), ten-point height of surface topography (Sz), skewness of topography height distribution (Ssk), surface bearing index (Sbi), kurtosis of topography height distribution (Sku), and RMS slope of the surface $(S \Delta q)$ are believed to have close relationship with some kinds of tribological properties. Thus, the problem of the validation of using fractal parameters to characterizing tribological properties emerges.

In this paper, the probability distributions of the traditional parameters mentioned above of 50 synthesized same-parameter fractal surfaces were studied; two basic tribological properties, the maximum temperature and contact area on friction surface in sliding process, were obtained by finite element analysis (FEA); and a discussion were performed at last.

\section{Fractal Surface Synthesis}

In general, fractal surfaces synthesized by computer algorithm, such as random midpoint displacement method (RMD), are not stable, which leads to all traditional parameters of synthesized surfaces be different every time. In this paper, the synthesis algorithm used was based on inverse discrete Fourier transform (DFT) proposed in Ref. [6] that guarantees the stability of synthesized fractal surfaces in which all the value of $\mathrm{Sq}$ are the same with given synthesis parameters $(C, D$, and sample numbers). When the sample numbers were assumed to be the same along the two directions, Sq can be calculated by [6]

$$
S q=\frac{2}{N} \sqrt{C \sum_{u=0}^{N / 2-1} \sum_{v=0}^{N / 2-1} \frac{1}{\left(u^{2}+v^{2}\right)^{(4-D)}}}
$$

where $\mathrm{N}$ represents the number of sample points along one direction, for sake of fast calculation, $\mathrm{N}$ often equals an integer power of $2 ; \mathrm{u}, \mathrm{v}$ are sample point number in frequency domain. When $\mathrm{N}=64, \mathrm{D}=2.6$, $\mathrm{C}=4.1457 \times 10^{7}$, according to equation (1), Sq=5. Figure 1 shows a synthesized fractal surface.

\footnotetext{
${ }^{\mathrm{a}}$ Corresponding author: zhouc@fzu.edu.cn
} 


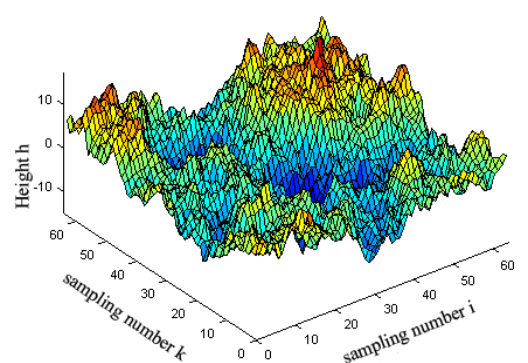

Figure 1. Synthesized fractal surface

The energy of a surface was defined as the summation of square of every sampling point's height, which is just the same as the definition of signal energy in signal processing $[7,8]$. It is generally known that FEA requires its geometric model to be smooth enough to avoid fatal errors in calculation. Thus, all surfaces used in this paper had been filtered by the method proposed in Ref. [6] to get rid of high frequency components before FEA.

$\mathrm{Sq}$ is defined as (the sampling points in two directions were assumed to be the same) [9]

$$
S q=\sqrt{\frac{1}{N^{2}} \sum_{j=1}^{N} \sum_{i=1}^{N} h^{2}(i, j)}
$$

where $h(i, j)$ represent the surface height in sampling point (i, j). With that definition, the Sq of a filtered surface can be calculated if the Sq before filtering and the percentage of reserved energy are known (detailed discussions can be found in Ref. [6]).

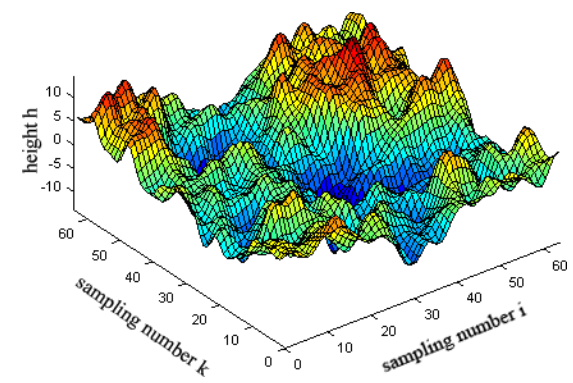

Figure 2. Filtered surface with $95 \%$ energy reserved

The synthesis parameters of 50 fractal surfaces used later were listed in table 1.

Table 1. Fractal surface synthesis parameters

\begin{tabular}{ccccc}
\hline $\begin{array}{c}\text { Sampling } \\
\text { number }\end{array}$ & $\begin{array}{c}\text { Scale } \\
\text { factor }\end{array}$ & $\begin{array}{c}\text { Fractal } \\
\text { dimension }\end{array}$ & $\begin{array}{c}\text { Sampling } \\
\text { interval } \\
(\mu \mathrm{m})\end{array}$ & $\begin{array}{c}\text { Percentage } \\
\text { of } \\
\text { reversed } \\
\text { energy }\end{array}$ \\
\hline $64 \times 64$ & $4.1457 \times 10^{7}$ & 2.6 & 2 & $95 \%$ \\
\hline
\end{tabular}

Since lubrication wasn't studied in this paper, and the definition of surface peak isn't consistent now, only Ssk, $\mathrm{Sku}, \mathrm{Sz}, \mathrm{Sbi}$, and $\mathrm{S} \Delta \mathrm{q}$ were chosen as traditional parameters to be discussed. The probability distributions of these parameters are shown in Figure 3, and table 2 listed the maximum, minimum, and average value of them.

Table 2. Maximum, minimum and average values of traditional parameters

\begin{tabular}{cccc}
\hline Parameter & Maximum & Minimum & Average \\
\hline Ssk & 1.2222 & -0.8151 & -0.1023 \\
$\mathrm{Sku}$ & 4.9672 & 2.2302 & 2.9058 \\
$\mathrm{Sz}$ & 34.8352 & 23.9845 & 27.9804 \\
$\mathrm{Sbi}$ & 2.1609 & 1.3007 & 1.6039 \\
$\mathrm{~S} \Delta \mathrm{q}$ & 0.8597 & 0.8040 & 0.8427 \\
\hline
\end{tabular}

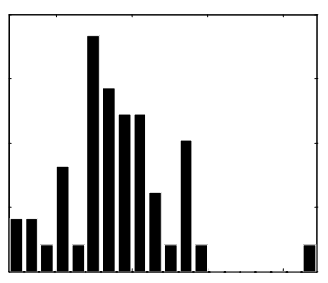

(a)

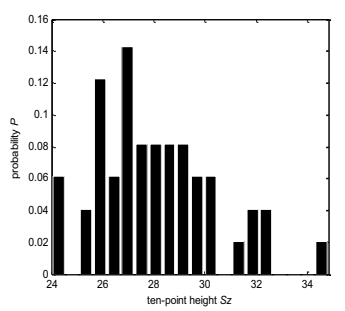

(c)

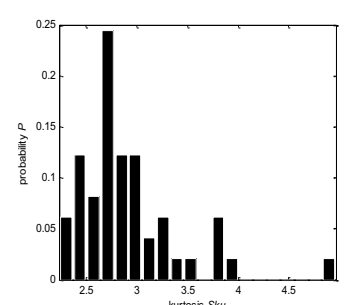

(b)

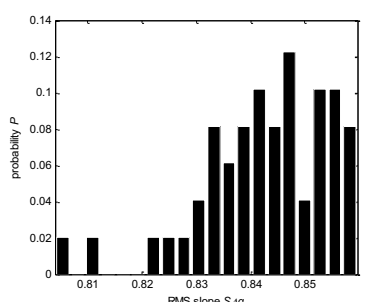

(d)

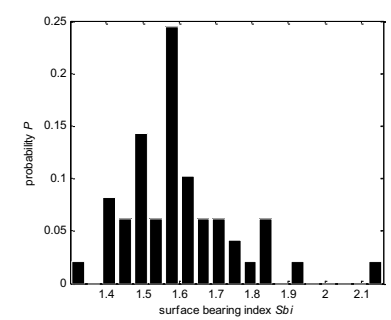

(e)

Figure 3. Probability distributions of traditional parameters

\section{FEA Modeling}

The FEA model of sliding process was composed of two components. One named "object A" shown in Figure 4 had a fractal rough surface synthesized by the algorithm mentioned above, and another one called "object B" was only a long rectangular plate which could be seen in Figure 5. Figure 5 also shows the geometric model of FEA where $\mathrm{L}=800 \mu \mathrm{m}, 11=150 \mu \mathrm{m}, \mathrm{e} 1=60 \mu \mathrm{m}, 12=126 \mu \mathrm{m}$, $\mathrm{e} 2=40+\mathrm{hmax}(\mu \mathrm{m})$, in which hmax is the distance from the highest summit to the mean plane.

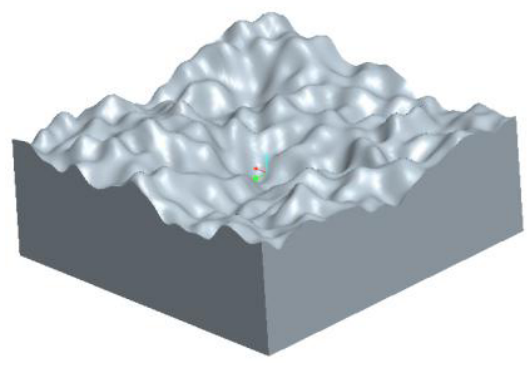

Figure 4. Object A with a fractal surface 


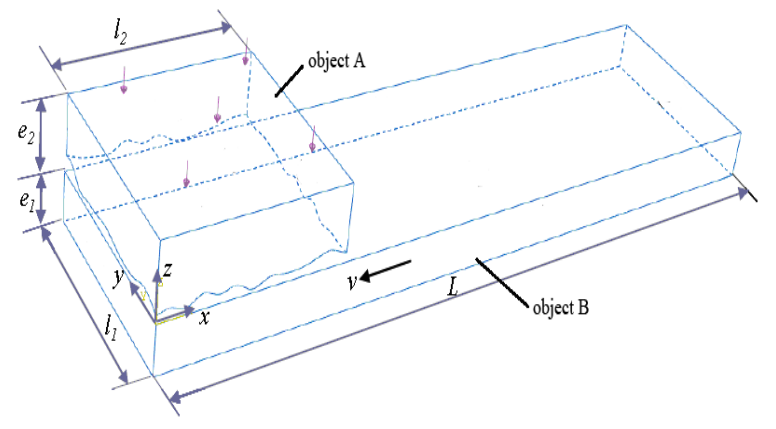

Figure 5. Geometric model of FEA

In the FEA process, object B was assumed to be rigid, and object A was a kind of steel. The material property of A was listed in table 3(Elastic modulus is 200GPa), and Figure 6 shows its true stress-strain curve. Firstly, all freedom of object B was fixed, and the load whose final value was $100 \mathrm{MPa}$ was applied linearly on the top surface of $A$ along the negative $Z$ direction within $5 \mu$ s. Then, when object A was fixed, object B was pushed to slide along the negative $X$ direction in the speed of $60 \mathrm{~m} / \mathrm{s}$ with friction coefficient equaled 0.2 .

Table 3. Material properties of object A

\begin{tabular}{ccccc}
\hline $\begin{array}{c}\text { Conductivity } \\
(\mathrm{W} \cdot \mathrm{m}-1 \cdot \mathrm{K}- \\
1)\end{array}$ & $\begin{array}{c}\text { Density } \\
(\mathrm{kg} \cdot \mathrm{m}- \\
3)\end{array}$ & $\begin{array}{c}\text { Specific } \\
\text { heat } \\
(\mathrm{J} \cdot \mathrm{kg}- \\
1 \cdot \mathrm{K}-1)\end{array}$ & $\begin{array}{c}\text { Thermal } \\
\text { expansion } \\
(\mathrm{K}-1)\end{array}$ & $\begin{array}{c}\text { Poisson's } \\
\text { ratio }\end{array}$ \\
\hline 48.15 & 7810 & 468.9 & 11.59 & 0.25 \\
\hline
\end{tabular}

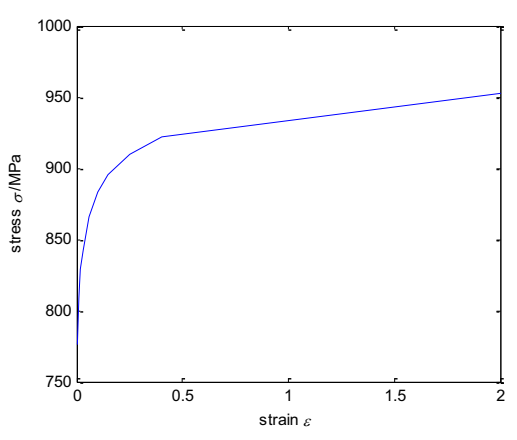

Figure 6. True stress-strain curve

\section{Results and Discussion}

The simulation results of one out of 50 samples were shown in figures below. Figure 7 is the thermal field distribution when the maximum temperature of object $\mathrm{A}$ reached its highest value. Figure 8 shows, in the whole sliding process, the value of the highest temperature in object $\mathrm{A}$. At the beginning, it surged, and about $6 \mu$ s later, it became stable and fluctuated around a certain value.

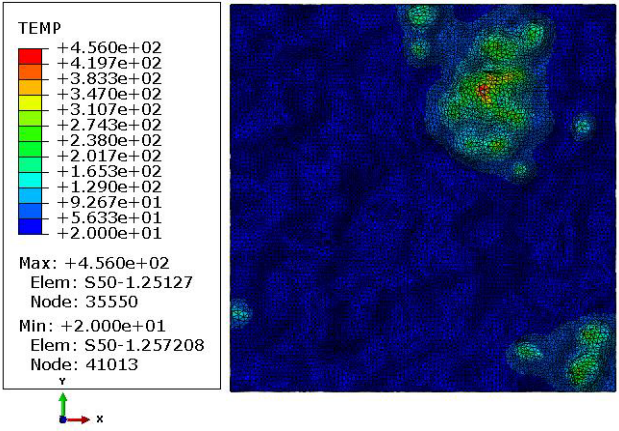

Figure 7. Thermal field distribution of object A

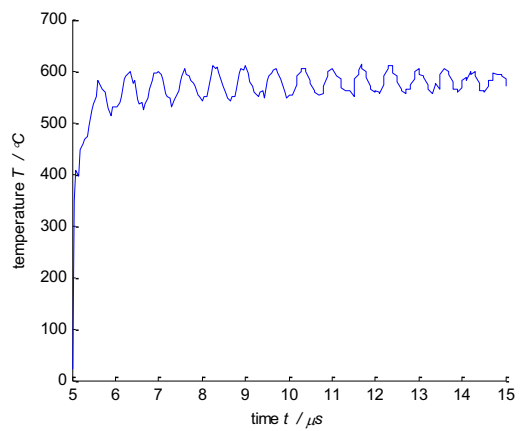

Figure 8. Change of maximum temperature in the whole sliding process

Figure 9 shows the change of contact area in the sliding process which fluctuated strongly in the early stage, then became stable after a short time.

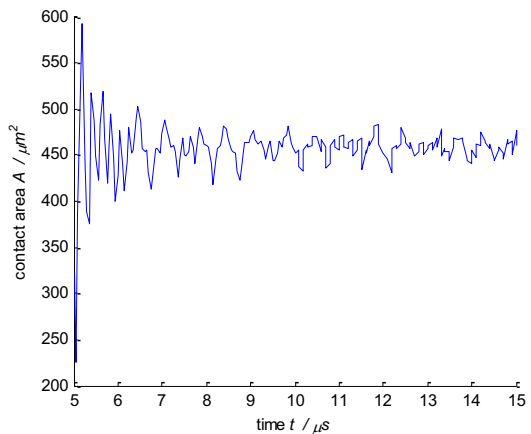

Figure 9. Change of contact area in the whole sliding process

Since the temperature in the friction pair affects a lot of tribological properties, and the contact area characterizes the bearing ability of components, the maximum temperature and contact area were studied in this article (in order to avoid the non-stable value in the early stage, all values were calculated after $8 \mu \mathrm{s}$ ). Because of the fluctuation of the value, the maximum temperature Tmax was defined as the average of the peak values during the evaluation time, and the contact area Aavg was the average of the values calculated.

Figure 10 represents the probability distribution of the Tmax and Aavg. It tells that the values calculated changed largely though the fractal surfaces were synthesized by the same parameters, which means the tribological properties of these samples are different. 


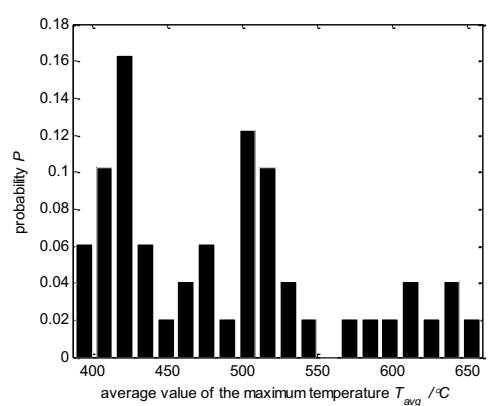

(a)

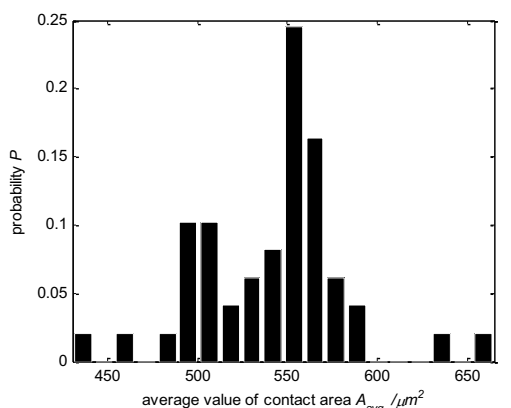

(b)

Figure 10. Maximum temperature and average area of surfaces

From the results obtained, the fractal parameters may be a much better way to represent the selfsimilarity/affinity of rough surfaces, but still not good enough to characterize the tribological properties. Certainly, traditional parameters are not good enough to characterize tribological properties as thought, too. In the insight of the relation between Ssk, Sku, Sz, Sbi, S $\Delta \mathrm{q}$ and Aavg (shown in Figure 11), their relationship are too complex to get a regular conclusion, which may reflects the "chaotic" property in tribology (the relationship between Ssk, Sku, Sz, Sbi, S $\Delta \mathrm{q}$ and Tmax show the same situation, too).

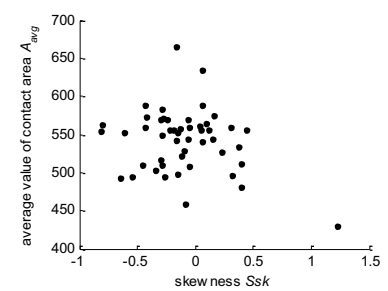

(a)

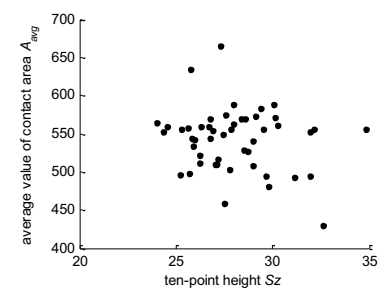

(c)

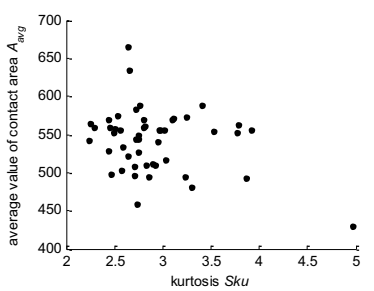

(b)

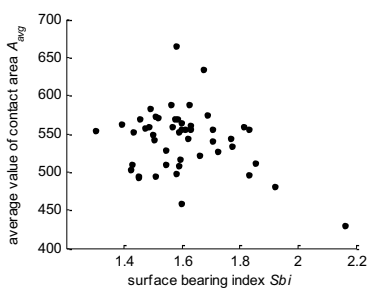

(d)

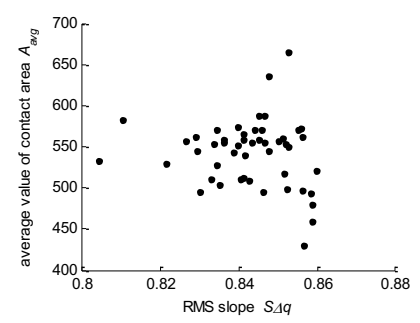

(e)

Figure 11. Relationship between traditional parameters and contact area

\section{CONCLUSIONS}

The major results of the present work can be summarized in the following points:

1. Fractal surfaces are characterized by fractal dimension D and scale factor C. Although one kind of special computer algorithm can synthesize stable fractal surfaces which have prescribed $\mathrm{Sq}$, other traditional parameters such as Ssk, Sku, Sz, Sbi, S $\Delta \mathrm{q}$ are still random.

2.Two basic tribological properties, maximum temperature and contact area, calculated by FEA changed dramatically, which told the fractal parameters may not be good to characterize tribological properties.

3. The relationship between tribological properties and traditional parameters are obscure, too.

From results mentioned above, tribological properties are not easy to characterize by some simple parameters, studies following should be focus on some other geometric feature of surfaces, for example surface texture, and the simplified FEA model may also affect the validation of this study, a more reasonable one should be proposed later.

Tribological properties. Certainly, traditional parameters are not good enough to characterize tribological properties as thought, too. In the insight of the relation between Ssk, Sku, Sz and Aavg (shown in Figure 5), their relationship are too complex to get a regular conclusion, which may reflects the "chaotic" property in tribology (the relationship between Ssk, Sku, $\mathrm{Sz}$ and Tmax show the same situation, too).

\section{ACKNOWLEDGEMENT}

This project was financially supported by Fujian Provincial Natural Science Foundation of China(Grant No. 2012J01206).

\section{REFERENCE}

[1] Zhang, S. J., et al. "A review of surface roughness generation in ultra-precision machining." Int. J. Mach. Tool. Manu 91,(2015).

[2] Nunez, Emerson Escobar, and A. A. Polycarpou. "The effect of surface roughness on the transfer of polymer Films under unlubricated testing Conditions." Wear,326-327,(2015). 
[3] Majumdar, A., and C. L. Tien. "Fractal characterization and simulation of rough surfaces." Wear, 136.90(1990).

[4] Ozer, A., and H. Sofuoglu. "Thermo-mechanical analysis of the magnetic head-disk interface with a fractal surface description." Wear,266.11-12(2009).

[5] Vallet, C., et al. "Real versus synthesized fractal surfaces: Contact mechanics and transport properties." Tribol. Int.,142,(2009).
[6] ZHOU, and GAO. "Fractal Surface Synthesis Based on Two Dimensional Discrete Fourier Transform." Chin. J. Chem. Eng..6(2013).

[7] R. C. Gonzalez, R. E. Woods. Digital image processing. 2nd ed. New Jersey: Prentice Hall, 2002.

[8] K. R. Castleman. Digital image processing. New Jersey: Prentice Hall, 1996. 